九州大学学術情報リポジトリ

Kyushu University Institutional Repository

\title{
RATE OF CONVERGENCE IN DISTRIBUTION OF A LINEAR COMBINATION OF \$ U \$-STATISTICS FOR A DEGENERATE KERNEL
}

Yamato, Hajime

Department of Mathematics and Computer Science, Kagoshima University

Kondo, Masao

Department of Mathematics and Computer Science, Kagoshima University

https://doi.org/10.5109/13509

出版情報: Bulletin of informatics and cybernetics. 34 (1), pp.61-73, 2002-10. Research Association of Statistical Sciences

バージョン :

権利関係 : 


\title{
RATE OF CONVERGENCE IN DISTRIBUTION OF A LINEAR COMBINATION OF U-STATISTICS FOR A DEGENERATE KERNEL
}

\author{
By \\ Hajime YAMATO* and Masao KONDO'
}

\begin{abstract}
Associated with an estimable parameter, we consider a linear combination of U-statistics (Toda and Yamato, 2001) which includes V-statistic and LB-statistic. For a degenerate kernel, its asymptotic distribution (Yamato et al., 2001) is easily derived by the same method as Yamato and Toda (2001). We give the rate of this convergence in distribution.
\end{abstract}

Key Words and Phrases: Linear combination of U-statistics, order of degeneracy, rate of convergence.

\section{Introduction}

Let $\theta(F)$ be an estimable parameter of an unknown distribution function $F$ which has a symmetric kernel $g\left(x_{1}, \ldots, x_{k}\right)$ of degree $k(\geq 2)$ and $X_{1}, \ldots, X_{n}$ be a random sample of size $n$ from $F$.

As an estimator of $\theta(F)$, Toda and Yamato (2001) introduced a linear combination $Y_{n}$ of U-statistics as follows. Let $w\left(r_{1}, \ldots, r_{j} ; k\right)$ be a nonnegative and symmetric function of positive integers $r_{1}, \ldots, r_{j}$ such that $j=1, \ldots, k$ and $r_{1}+\cdots+r_{j}=k$, where $k$ is the degree of the kernel $g$ and fixed. We assume that at least one of $w\left(r_{1}, \ldots, r_{j} ; k\right)$ 's is positive. For $j=1, \ldots, k$, let $g_{(j)}\left(x_{1}, \ldots, x_{j}\right)$ be the kernel given by

$$
g_{(j)}\left(x_{1}, \ldots, x_{j}\right)=\frac{1}{d(k, j)} \sum_{r_{1}+\cdots+r_{j}=k}^{+} w\left(r_{1}, \ldots, r_{j} ; k\right) g\left(x_{1}^{r_{1}}, \ldots, x_{j}^{r_{j}}\right)
$$

where

$$
g\left(x_{1}^{r_{1}}, \ldots, x_{n}^{r_{n}}\right)=g(\underbrace{x_{1}, \ldots, x_{1}}_{r_{1}}, \ldots, \underbrace{x_{n}, \ldots, x_{n}}_{r_{n}})
$$

and the summation $\sum_{r_{1}+\cdots+r_{j}=k}^{+}$is taken over all positive integers $r_{1}, \ldots, r_{j}$ satisfying $r_{1}+\cdots+r_{j}=k$ with $j$ and $k$ fixed and $d(k, j)=\sum_{r_{1}+\cdots+r_{j}=k}^{+} w\left(r_{1}, \ldots, r_{j} ; k\right)$ for $j=1,2, \ldots, k$.

Let $U_{n}^{(j)}$ be the U-statistic associated with this kernel $g_{(j)}\left(x_{1}, \ldots, x_{j} ; k\right)$ for $j=$

\footnotetext{
* Department of Mathematics and Computer Science, Kagoshima University, Kagoshima 890-0065, Japan.

$\dagger$ Department of Mathematics and Computer Science, Kagoshima University, Kagoshima 890-0065, Japan.
} 
$1, \ldots, k$. The kernel $g_{(j)}\left(x_{1}, \ldots, x_{j} ; k\right)$ is symmetric because of the symmetry of $w\left(r_{1}, \ldots\right.$, $\left.r_{j} ; k\right)$. If $d(k, j)$ is equal to zero for some $j$, then the associated $w\left(r_{1}, \ldots, r_{j} ; k\right)$ 's are equal to zero. In this case, we let the corresponding statistic $U_{n}^{(j)}$ be zero. The statistics $Y_{n}$ is given by

$$
Y_{n}=\frac{1}{D(n, k)} \sum_{j=1}^{k} d(k, j)\left(\begin{array}{l}
n \\
j
\end{array}\right) U_{n}^{(j)}
$$

where $D(n, k)=\sum_{j=1}^{k} d(k, j)\left(\begin{array}{l}n \\ j\end{array}\right)$. Since $w$ 's are nonnegative and at least one of them is positive, $D(n, k)$ is positive.

$Y_{n}$ generalizes well-known statistics. Four examples are given to show this. First, let $w$ be the function given by $w(1,1, \ldots, 1 ; k)=1$ and $w\left(r_{1}, \ldots, r_{j} ; k\right)=0$ for positive integers $r_{1}, \ldots, r_{j}$ such that $j=1, \ldots, k-1$ and $r_{1}+\cdots+r_{j}=k$. Then $Y_{n}$ is equal to $\mathrm{U}$-statistic $U_{n}$, which is given by

$$
U_{n}=\left(\begin{array}{l}
n \\
k
\end{array}\right)^{-1} \sum_{1 \leq j_{1}<\cdots<j_{k} \leq n} g\left(X_{j_{1}}, \ldots, X_{j_{k}}\right),
$$

where $\sum_{1 \leq j_{1}<\ldots<j_{k} \leq n}$ denotes the summation over all integers $j_{1}, \ldots, j_{k}$ satisfying $1 \leq$ $j_{1}<\cdots<j_{k} \leq n$.

Second, let $w$ be the function given by $w\left(r_{1}, \ldots, r_{j} ; k\right)=1$ for positive integers $r_{1}, \ldots, r_{j}$ such that $j=1, \ldots, k$ and $r_{1}+\cdots+r_{j}=k$. Then $Y_{n}$ is equal to the LBstatistic $B_{n}$ given by

$$
B_{n}=\left(\begin{array}{c}
n+k-1 \\
k
\end{array}\right)^{-1} \sum_{r_{1}+\cdots+r_{n}=k} g\left(X_{1}^{r_{1}}, \ldots, X_{n}^{r_{n}}\right),
$$

where $\sum_{r_{1}+\cdots+r_{n}=k}$ denotes the summation over all non-negative integers $r_{1}, \ldots, r_{n}$ satisfying $r_{1}+\cdots+r_{n}=k$.

Third, let $w$ be the function given by $w\left(r_{1}, \ldots, r_{j} ; k\right)=k ! /\left(r_{1} ! \cdots r_{j} !\right)$ for positive integers $r_{1}, \ldots, r_{j}$ such that $j=1, \ldots, k$ and $r_{1}+\cdots+r_{j}=k$. In case of $k=3, Y_{n}$ is equal to the V-statistic $V_{n}$ given by

$$
V_{n}=\frac{1}{n^{k}} \sum_{j_{1}=1}^{n} \cdots \sum_{j_{k}=1}^{n} g\left(X_{j_{1}}, \ldots, X_{j_{k}}\right) .
$$

(See Toda and Yamato, 2001).

The last, let $w$ be the function given by $w\left(r_{1}, \ldots, r_{j} ; k\right)=k ! /\left(r_{1} \cdots r_{j}\right)$ for positive integers $r_{1}, \ldots, r_{j}$ such that $j=1, \ldots, k$ and $r_{1}+\cdots+r_{j}=k$. In the case of $k=3, Y_{n}$ for the third central moment of the distribution $F$ is given by

$$
S_{n}=\frac{n}{n^{2}+1} \sum_{i=1}^{n}\left(X_{i}-\bar{X}\right)^{3}
$$

where $\bar{X}$ is the sample mean of $X_{1}, \ldots, X_{n}$ (see Nomachi et al., 2002).

Now, for the kernel $g\left(x_{1}, \ldots, x_{k}\right)$, we put

$$
\psi_{j}\left(x_{1}, \ldots, x_{j}\right)=E\left[g\left(X_{1}, \ldots, X_{k}\right) \mid X_{1}=x_{1}, \ldots, X_{j}=x_{j}\right], \quad j=1, \ldots, k
$$


and

$$
\sigma_{j}^{2}=\operatorname{Var}\left[\psi_{j}\left(X_{1}, \ldots, X_{j}\right)\right], \quad j=1, \ldots, k
$$

In this paper, we suppose that

$$
\sigma_{1}^{2}=\cdots=\sigma_{d-1}^{2}=0 \text { and } \sigma_{d}^{2}>0,
$$

that is, the U-statistic and/or the kernel $g$ is degenerate of order $d-1$. Hence, $E \psi_{d}\left(X_{1}\right.$, $\left.\ldots, X_{d}\right)=\theta$ and with probability one (w.p.1) $\psi_{1}\left(X_{1}\right)=\theta, \ldots, \psi_{d-1}\left(X_{1}, \ldots, X_{d-1}\right)=\theta$.

The asymptotic distribution of $U_{n}$ was studied by Lee (1990), Koroljuk and Borovskich (1994) and Borovskich (1996). We may summarize their results as follows. Let $W$ be the Gaussian random measure associated with the distribution $F$ on the real line $(-\infty, \infty)$ such that $E W(A)=0$ and $E W(A) W(B)=F(A \cap B)$ for any Borel sets $A$, $B$. Denote the stochastic integral of the function $f\left(x_{1}, \ldots, x_{c}\right)$ by $J_{c}(f)$, that is,

$$
J_{c}(f)=\int_{-\infty}^{\infty} \cdots \int_{-\infty}^{\infty} f\left(x_{1}, \ldots, x_{c}\right) W\left(d x_{1}\right) \cdots W\left(d x_{c}\right) .
$$

Alternatively, $J_{c}(f)$ can be represented by using an orthonormal basia $e_{1}, e_{2}, \ldots$ of $L_{2}(F)$. For any function $f_{1}$ and $f_{2}$ such that $\int_{R^{c}} f_{i}\left(x_{1}, \ldots, x_{c}\right)^{2} \prod_{j=1}^{c} d F\left(x_{j}\right)<\infty(i=1,2)$, their inner product is given by $\left(f_{1}, f_{2}\right)=\int_{R^{c}} f_{1}\left(x_{1}, \ldots, x_{c}\right) f_{2}\left(x_{1}, \ldots, x_{c}\right) \prod_{j=1}^{c} d F\left(x_{j}\right)$. $J_{c}(f)$ is also written as

$$
J_{c}(f)=\sum_{i_{1}=1}^{\infty} \cdots \sum_{i_{c}=1}^{\infty}\left(f, e_{i_{1}} \cdots e_{i_{c}}\right) \prod_{l=1}^{\infty} H_{r_{l}(\mathbf{i})}\left(Z_{l}\right),
$$

where $H_{r}$ is the $r$-th Hermite polynomial, $\left\{Z_{l}\right\}_{l=1}^{\infty}$ is a sequence of independent standard normal random variables and $r_{l}(\mathbf{i})$ is the number of indices among $\mathbf{i}=\left(i_{1}, \ldots, i_{d}\right)$ equal to $l$. For the degenerate kernel $g$ of order $d-1$, under the conditions $E\left|g\left(X_{1}, \ldots, X_{k}\right)\right|^{2}<$ $\infty$, the asymptotic distribution of $U_{n}$ is given by

$$
n^{d / 2}\left(U_{n}-\theta\right) \stackrel{\mathcal{D}}{\rightarrow}\left(\begin{array}{l}
k \\
d
\end{array}\right) J_{d}\left(\xi_{d, d}\right),
$$

where $\stackrel{\mathcal{D}}{\rightarrow}$ means the convergence in distribution as $n \rightarrow \infty$ and $\xi_{d, d}\left(x_{1}, \ldots, x_{d}\right)=$ $\psi_{d}\left(x_{1}, \ldots, x_{d}\right)-\theta$.

The convergence (1.6) also holds under the following conditions (i) or (ii): (i) $E\left|g^{(c)}\left(X_{1}, \ldots, X_{d}\right)\right|^{2 c /(2 c-d)}<\infty$ for $c=d, d+1, \ldots, k$ (see Koroljuk and Borovskich,1994). (ii) $E\left|g^{(d)}\left(X_{1}, \ldots, X_{d}\right)\right|^{2}<\infty$ and $t^{2 c /(2 c-d)} P\left[\left|g^{(c)}\right|>t\right] \rightarrow 0(t \rightarrow \infty)$ for $c=d+1, \ldots, k$ (see Borovskich,1996).

Yamato et al. (2001) studied an invariance principle (functional limit theorem) of $Y_{n}$ and derived its asymptotic distribution as a special case of the principle. Also the asymptotic distribution of $Y_{n}$ may be obtained by the method of Yamato and Toda (2001).

The purpose of this paper is to give the asymptotic distribution of $Y_{n}$ directly by the later method and to evaluate the rate of its convergence, for the degenerate kernel. In Section 2, we give the asymptotic distribution of a linear combination $Y_{n}$ of U-statistics given by (1.2). In Section 3, we give its rate of convergence in distribution. 


\section{Asymptotic distribution of $Y$-statistic}

For kernel $g_{(j)}\left(x_{1}, \ldots, x_{j}\right)$ given by $(1.1)$, we put for $c=1, \ldots, j$ and $j=1, \ldots, k$

$$
\begin{aligned}
& \psi_{(j), c}\left(x_{1}, \ldots, x_{c}\right)=E\left[g_{(j)}\left(X_{1}, \ldots, X_{j}\right) \mid X_{1}=x_{1}, \ldots, X_{c}=x_{c}\right] \\
& =\frac{1}{d(k, j)} \sum_{r_{1}+\cdots+r_{j}=k}^{+} w\left(r_{1}, \ldots, r_{j} ; k\right) E g\left(x_{1}^{r_{1}}, \ldots, x_{c}^{r_{c}}, X_{c+1}^{r_{c+1}}, \ldots, X_{j}^{r_{j}}\right),
\end{aligned}
$$

where on the right-hand side we use the notation used in (1.1).

The U-statistic $U_{n}^{(j)}, j=1, \ldots, k$ corresponding to to the kernel $g_{(j)}$ have the following properties.

Lemma 2.1. (Yamato et al., 2001)

$$
E\left[U_{n}^{(j)}\right]=\theta, \quad k-\frac{d-1}{2} \leq j \leq k
$$

or

$$
E\left[U_{n}^{(k-j)}\right]=\theta, \quad 0 \leq j \leq \frac{d-1}{2}
$$

LEMMA 2.2. (Yamato et al., 2001) The order of degeneracy of $U_{n}^{(k-j)}$ is at least $d-2 j-1$ for $1 \leq j \leq(d-1) / 2$ and

$$
\psi_{(k-j), d-2 j}\left(x_{1}, \ldots, x_{d-2 j}\right)=\theta+\left(\begin{array}{c}
k-d+j \\
j
\end{array}\right) \frac{w\left(1^{k-2 j}, 2^{j} ; k\right)}{d(k, k-j)}\left[\varphi_{d, d-2 j}\left(x_{1}, \ldots, x_{d-2 j}\right)-\theta\right]
$$

where for $1 \leq j \leq(d-1) / 2$

$$
\varphi_{d, d-2 j}\left(x_{1}, \ldots, x_{d-2 j}\right)=E\left[\psi_{d}\left(x_{1}, \ldots, x_{d-2 j}, X_{d-2 j+1}, X_{d-2 j+1}, \ldots, X_{d-j}, X_{d-j}\right)\right]
$$

and

$$
w\left(1^{r}, 2^{s} ; k\right)=w(\underbrace{1,1, \cdots, 1}_{r}, \underbrace{2,2, \cdots, 2}_{s} ; k) .
$$

By the similar method to the above Lemma, we can show that for $1 \leq j \leq(d-1) / 2$,

$$
E \varphi_{d, d-2 j}\left(X_{1}, \ldots, X_{d-2 j}\right)=\theta .
$$

From Lemmas 2.1 and 2.2, it follows that If $d=2 l+1$ and $l$ is a positive integer, then $E U_{n}^{(k)}=E U_{n}^{(k-1)}=\cdots=E U_{n}^{(k-l+1)}=E U_{n}^{(k-l)}=\theta$. The orders of degeneracy of $U_{n}^{(k-1)}, \ldots, U_{n}^{(k-l+1)}, U_{n}^{(k-l)}$ are at least $2(l-1), \ldots, 2,0$, respectively. If $d=2 l$ and $l$ is a positive integer, then $E U_{n}^{(k)}=E U_{n}^{(k-1)}=\cdots=E U_{n}^{(k-l+2)}=E U_{n}^{(k-l+1)}=$ $\theta$. The orders of degeneracy of $U_{n}^{(k-1)}, \ldots, U_{n}^{(k-l+2)}, U_{n}^{(k-l+1)}$ are at least $2 l-3, \ldots, 3,1$, respectively.

Lemma 2.3. (Yamato et al., 2001) In case of $d=2 l$,

$$
E U_{n}^{(k-l)}-\theta=\frac{1}{d(k, k-l)}\left(\begin{array}{c}
k-l \\
k-d
\end{array}\right) w\left(1^{k-d}, 2^{l} ; k\right)\left[E \psi_{d}\left(X_{1}, X_{1}, \ldots, X_{l}, X_{l}\right)-\theta\right] .
$$


Now we assume $d(k, k)=w(1, \ldots, 1 ; k)>0$, then, it follows that $U_{n}^{(k)}=U_{n}$, because of $g_{(k)}=g$. Under this assumption, there exists a constant $b(\geq 0)$ such that

$$
\frac{d(k, k) n^{(k)}}{D(n, k) k !}=1-\frac{b}{n}+O\left(\frac{1}{n^{2}}\right)
$$

Thus

$$
\frac{n^{k}}{D(n, k)} \rightarrow \frac{k !}{d(k, k)} \text { as } n \rightarrow \infty \text {. }
$$

For the U-statistic $U_{n}, d(k, k) n^{(k)} /[D(n, k) k !]=1$. For the V-statistic $V_{n}$ and the Sstatistic $S_{n}, b=k(k-1)$. For the LB-statistic $B_{n}, b=k(k-1) / 2$. (see Nomachi et al., 2001)). It is assumed that $n^{k} / D(n, k)$ is nondecreasing in Yamato et al. (2001) for invariance principle, but the convergence in distribution needs only the convergence (2.6).

Yamato et al. (2001) obtained the asymptotic distribution of $Y_{n}$ from invariance principle. Alternatively, it can be obtained directly by the same method as Yamato and Toda (2001). We state here the idea of how to compute directly the asymptotic distribution of $Y_{n}$. Noting the degeneracy of $U_{n}^{(k-j)}, 0 \leq j \leq(d-1) / 2$, stated above, we expand $Y_{n}$ as follows: For $d=2 l+1$

$$
n^{d / 2}\left(Y_{n}-\theta\right)=\sum_{j=0}^{l} T_{j, n}+R_{1 n}
$$

where

$$
\begin{gathered}
T_{j, n}=d(k, k-j) \frac{\left(\begin{array}{c}
n \\
k-j
\end{array}\right)}{D(n, k)} n^{d / 2}\left(U_{n}^{(k-j)}-\theta\right), j=0,1, \ldots, l, \\
R_{1 n}=\sum_{j=1}^{k-l-1} d(k, j) \frac{\left(\begin{array}{l}
n \\
j
\end{array}\right)}{D(n, k)} n^{d / 2}\left(U_{n}^{(j)}-\theta\right) .
\end{gathered}
$$

By Minkowski's inequality we have

$$
\left[E R_{1 n}^{2}\right]^{1 / 2} \leq \sum_{j=1}^{k-l-1} d(k, j) \frac{\left(\begin{array}{l}
n \\
j
\end{array}\right)}{D(n, k)} n^{d / 2}\left[E\left(U_{n}^{(j)}-\theta\right)^{2}\right]^{1 / 2} .
$$

By the relation (2.6), $\left(\begin{array}{l}n \\ j\end{array}\right) n^{d / 2} / D(n, k) \sim c n^{j-k+d / 2}(n \rightarrow \infty)$, where $c$ is a positive constant. For $1 \leq j \leq k-l-1$, it hols that $j-k+d / 2 \leq-l-1+d / 2 \leq-1 / 2$. Under the condition (2.11) given in the following theorem 2.4, we have $E\left(U_{n}^{(j)}-\theta\right)^{2}<\infty$ and therefore it follows that $E R_{1 n}^{2}=O\left(n^{-1}\right)$. In case of $d=2 l$, it holds that $j-k+d / 2 \leq$ $-l-1+d / 2 \leq-1$ for $1 \leq j \leq k-l-1$. Thus, for $d=2 l$,

$$
n^{d / 2}\left(Y_{n}-\theta\right)=\sum_{j=0}^{l-1} T_{j, n}+T_{l, n}+R_{2 n}
$$

where $E R_{2 n}^{2}=O\left(n^{-2}\right)$ under the condition (2.11) given in the following theorem 2.4. $R_{1 n}, R_{2 n}$ do not affect the convergence in distribution of $n^{d / 2}\left(Y_{n}-\theta\right)$. 
We note that $g_{(k)}=g$ and the kernel $g$ is assumed to be degenerate of order $d-1$. We put $g_{(k)}^{(d)}\left(x_{1}, \ldots, x_{d}\right)=\psi_{d}\left(x_{1}, \ldots, x_{d}\right)-\theta\left(=\varphi_{d, d}\left(x_{1}, \ldots, x_{d}\right)-\theta\right)$. Then, by (1.6) it follows

$$
n^{d}\left(U_{n}^{(k)}-\theta\right) \stackrel{\mathcal{D}}{\rightarrow}\left(\begin{array}{l}
k \\
d
\end{array}\right) J\left(g_{(k)}^{(d)}\right) .
$$

Similarly, noting the degeneracy of the kernel $g_{(k-j)}$ given by Lemma 2.2, we put

$$
g_{(k-j)}^{(d-2 j)}\left(x_{1}, \ldots, x_{d-2 j}\right)=\psi_{(k-j), d-2 j}\left(x_{1}, \ldots, x_{d-2 j}\right)-\theta, \quad 1 \leq j \leq(d-1) / 2 .
$$

For $1 \leq j \leq(d-1) / 2$, at first we assume that the order of degeneracy of $U_{n}^{(k-j)}$ is $d-2 j-1$. Then, by Lemma 2.1 and (1.6), it follows that

$$
n^{d-2 j}\left(U_{n}^{(k-j)}-\theta\right) \stackrel{\mathcal{D}}{\rightarrow}\left(\begin{array}{c}
k-j \\
d-2 j
\end{array}\right) J_{d-2 j}\left(g_{(k-j)}^{(d-2 j)}\right), \quad 1 \leq j \leq(d-1) / 2 .
$$

By $(2.1)$, for $1 \leq j \leq(d-1) / 2$

$$
g_{(k-j)}^{(d-2 j)}\left(x_{1}, \ldots, x_{d-2 j}\right)=\frac{1}{d(k, k-j)}\left(\begin{array}{c}
k-d+j \\
j
\end{array}\right) w\left(1^{k-2 j}, 2^{j} ; k\right) \xi_{d, d-2 j}\left(x_{1}, \ldots, x_{d-2 j}\right),
$$

where

$$
\xi_{d, d-2 j}\left(x_{1}, \ldots, x_{d-2 j}\right)=\varphi_{d, d-2 j}\left(x_{1}, \ldots, x_{d-2 j}\right)-\theta .
$$

If for some $j_{0}\left(1 \leq j_{0} \leq(d-1) / 2\right)$ the order of degeneracy of $U_{n}^{\left(k-j_{0}\right)}$ is less than $d-2 j_{0}-1$, then the limit in distribution of $U_{n}^{\left(k-j_{0}\right)}$ vanish because of $g_{\left(k-j_{0}\right)}^{\left(d-2 j_{0}\right)}=0$. In the case of $d=2 l, T_{l, n}$ converges to a constant as $n \rightarrow \infty$. Applying these convergence of $U_{n}^{(k-j)}(0 \leq j \leq(d-1) / 2)$ to $(2.7)$ and $(2.8)$, we get the following proposition.

THEOREM 2.4. (Yamato et al., 2001) We suppose that

$$
E\left[g\left(X_{j_{1}}, X_{j_{2}}, \ldots, X_{j_{k}}\right)^{2}\right]<\infty
$$

for all $j_{1}, j_{2}, \ldots, j_{k}$ such that $1 \leq j_{1} \leq j_{2} \leq \cdots \leq j_{k} \leq k$. We assume $d(k, k)>0$. Then in case of $d=2 l+1(l=1,2, \ldots)$, we have

$$
n^{d / 2}\left(Y_{n}-\theta\right) \stackrel{\mathcal{D}}{\rightarrow} \sum_{j=0}^{l} \tau_{j}
$$

where

$$
\tau_{j}=\frac{k !}{(k-d) !} \frac{1}{(d-2 j) ! j !} \cdot \frac{w\left(1^{k-2 j}, 2^{j} ; k\right)}{w\left(1^{k} ; k\right)} J_{d-2 j}\left(\xi_{d, d-2 j}\right), \quad j=0,1, \ldots, l
$$

and

$$
\xi_{d, d-2 j}=\varphi_{d, d-2 j}\left(x_{1}, \ldots, x_{d-2 j}\right)-\theta, j=0,1, \ldots, l .
$$

In case of $d=2 l(l=1,2, \ldots)$ we have

$$
n^{d / 2}\left(Y_{n}-\theta\right) \stackrel{\mathcal{D}}{\rightarrow} \sum_{j=0}^{l-1} \tau_{j}+\alpha_{l}
$$


where

$$
\alpha_{l}=\frac{k ! w\left(1^{k-d}, 2^{l} ; k\right)}{(k-d) ! l ! w\left(1^{k} ; k\right)}\left[E \psi_{d}\left(X_{1}, X_{1}, \ldots, X_{l}, X_{l}\right)-\theta\right] .
$$

The value $w\left(1^{k-2 j}, 2^{j} ; k\right) / w\left(1^{k} ; k\right)$ is equal to $1 / 2^{j}$ for the V-statistic $V_{n}$ and the Sstatistic $S_{n}$, and 1 for the LB-statistic $B_{n}$, respectively. The asymptotic distributions of the V-statistic $V_{n}$ and the LB-statistic $B_{n}$ are given by Yamato and Toda (2001) and Yamato et al. (2001). The asymptotic distribution of the S-statistic $S_{n}$ is given by the followings: In case of $d=2 l+1(l=1,2, \ldots)$, we have

$$
n^{d / 2}\left(S_{n}-\theta\right) \stackrel{\mathcal{D}}{\rightarrow} \frac{k !}{(k-d) !} \sum_{j=0}^{l} \frac{1}{(d-2 j) ! j ! 2^{j}} J_{d-2 j}\left(\xi_{d, d-2 j}\right) .
$$

In case of $d=2 l(l=1,2, \ldots)$ we have

$$
\begin{gathered}
n^{d / 2}\left(S_{n}-\theta\right) \stackrel{\mathcal{D}}{\rightarrow} \frac{k !}{(k-d) !}\left\{\sum_{j=0}^{l-1} \frac{1}{(d-2 j) ! j ! 2^{j}} J_{d-2 j}\left(\xi_{d, d-2 j}\right)\right. \\
\left.+\frac{1}{l ! 2^{l}}\left[E \psi_{d}\left(X_{1}, X_{1}, \ldots, X_{l}, X_{l}\right)-\theta\right]\right\} .
\end{gathered}
$$

\section{Rate of convergence}

To get the rate of convergence in distribution of $Y_{n}$, we use the following rate of convergence in distribution of the U-statistic $U_{n}$.

Lemma 3.1. (Theorem 6.5.2 of Borovskikh, 1996) Assume that

$$
\left|E \exp \left(i t J_{k}\left(\xi_{d, d}\right)\right)\right|=O\left(|t|^{-\gamma}\right)
$$

as $|t| \rightarrow \infty$, where $\gamma$ is some sufficiently large number. Then if $d$ is odd and

$$
E\left|g\left(X_{1}, \ldots, X_{k}\right)\right|^{3}<\infty
$$

it follows that

$$
\sup _{-\infty<t<\infty}\left|P\left(n^{d / 2}\left(U_{n}-\theta\right) \leq t\right)-P\left(\tau_{0} \leq t\right)\right|=O\left(n^{-\frac{1}{2}}\right) .
$$

Furthermore, if $d$ is even and

$$
E\left|g\left(X_{1}, \ldots, X_{k}\right)\right|^{4}<\infty
$$

then,

$$
\sup _{-\infty<t<\infty}\left|P\left(n^{d / 2}\left(U_{n}-\theta\right) \leq t\right)-P\left(\tau_{0} \leq t\right)\right|=o\left(n^{-\frac{1}{2}}\right) .
$$

This lemma is also given by Theorem 6.6.3 of Koroljuk and Borovskich (1994). Under the condition about the characteristic functions of Lemma 3.1, the corresponding distribution function has a bounded continuous derivative (see, for example, Corollary 11.6.1 of Kawata, 1972). 
THEOREM 3.2. We assume that $d(k, k)>0$ and

$$
\left|E \exp \left(i t J_{k-2 j}\left(\xi_{d, d-2 j}\right)\right)\right|=O\left(|t|^{-\gamma_{j}}\right), \quad 0 \leq j \leq(d-1) / 2
$$

as $|t| \rightarrow \infty$, where $\gamma_{j}$ is some sufficiently large number. Then if $d=2 l+1(l=1,2, \ldots)$ and

$$
E\left|g\left(X_{i_{1}}, \ldots, X_{i_{k}}\right)\right|^{3}<\infty \text { for } 1 \leq i_{1} \leq \cdots \leq i_{k} \leq k,
$$

it follows that

$$
\sup _{-\infty<t<\infty}\left|P\left(n^{d / 2}\left(Y_{n}-\theta\right) \leq t\right)-P\left(\sum_{j=0}^{l} \tau_{j} \leq t\right)\right|=O\left(n^{-\frac{1}{2}}\right) .
$$

If $d=2 l(l=1,2, \ldots)$ and

$$
E\left|g\left(X_{i_{1}}, \ldots, X_{i_{k}}\right)\right|^{4}<\infty \text { for } 1 \leq i_{1} \leq \cdots \leq i_{k} \leq k
$$

then, we have

$$
\sup _{-\infty<t<\infty}\left|P\left(n^{d / 2}\left(Y_{n}-\theta\right) \leq t\right)-P\left(\sum_{j=0}^{l-1} \tau_{j}+\alpha_{l} \leq t\right)\right|=O\left(n^{-\frac{1}{2}}\right) .
$$

In case of $d=2 l(l=1,2, \ldots)$, the rate of convergence given by (3.4) is weaker than (3.2). This is due to the evaluation of $l_{31}$ given later by (3.13). In order to prove this theorem, we need the following lemma.

LEMMA 3.3. For any random variables $W_{11}, W_{12}, W_{21}$ and $W_{22}$, we have

$$
\begin{gathered}
I_{11}=\sup _{-\infty<t<\infty}\left|P\left(W_{11}+W_{21} \leq t\right)-P\left(W_{12}+W_{22} \leq t\right)\right| \\
\leq \sup _{-\infty<t<\infty}\left|P\left(W_{11} \leq t\right)-P\left(W_{12} \leq t\right)\right|+\sup _{-\infty<t<\infty}\left|P\left(W_{21} \leq t\right)-P\left(W_{22} \leq t\right)\right| .
\end{gathered}
$$

We suppose that $W_{n} \stackrel{\mathcal{D}}{\rightarrow} W, E W_{n}^{2}$ is uniformly finite in $n=1,2, \ldots$, and that $a_{n}=1-(a / n)+O\left(n^{-2}\right)$, where $a$ is a constant. Then

$$
\begin{gathered}
I_{12}=\sup _{-\infty<x<\infty}\left|P\left(a_{n} W_{n} \leq x\right)-P(W \leq x)\right| \\
\leq \sup _{-\infty<x<\infty}\left|P\left(W_{n} \leq x\right)-P(W \leq x)\right|+O\left(\frac{1}{n}\right) .
\end{gathered}
$$

Proof of Lemma 3.3. For $I_{11}$, we have the following inequality.

$$
\begin{gathered}
I_{11} \leq \sup _{t}\left|E_{W_{21}}\left\{P\left(W_{11}+W_{21} \leq t \mid W_{21}\right)-P\left(W_{12}+W_{21} \leq t \mid W_{21}\right)\right\}\right| \\
+\sup _{t}\left|E_{W_{12}}\left\{P\left(W_{12}+W_{21} \leq t \mid W_{12}\right)-P\left(W_{12}+W_{22} \leq t \mid W_{12}\right)\right\}\right|
\end{gathered}
$$

where $E_{W}$ denotes the expectation with respect to the random variable $W$. The righthand side of the above inequality is less than or equal to

$$
E_{W_{21}} \sup _{t}\left|P\left(W_{11}+W_{21} \leq t \mid W_{21}\right)-P\left(W_{12}+W_{21} \leq t \mid W_{21}\right)\right|
$$




$$
+E_{W_{12}} \sup _{t}\left|P\left(W_{12}+W_{21} \leq t \mid W_{12}\right)-P\left(W_{12}+W_{22} \leq t \mid W_{12}\right)\right| .
$$

Thus we can obtain (3.5).

Next we shall prove the inequality (3.6). For $I_{12}$, we have

$$
I_{12} \leq \sup _{x}\left|P\left(a_{n} W_{n} \leq x\right)-P\left(W_{n} \leq x\right)\right|+\sup _{x}\left|P\left(W_{n} \leq x\right)-P(W \leq x)\right| .
$$

For any random variables $W$ and $\Delta$, it holds that

$$
\sup _{x}|P(W+\Delta \leq x)-P(W \leq x)| \leq 4(E|W \Delta|+E|\Delta|)
$$

(see p.261 of Shorack, 2000). Because $a_{n}=1-(a / n)+O\left(n^{-2}\right)$ and $E W_{n}^{2}$ is uniformly finite, we have from (3.7)

$$
\sup _{x}\left|P\left(a_{n} W_{n} \leq x\right)-P\left(W_{n} \leq x\right)\right| \leq 4\left[\frac{|a|}{n}\left(E W_{n}^{2}+E\left|W_{n}\right|\right)+O\left(\frac{1}{n^{2}}\right)\right]=O\left(\frac{1}{n}\right) .
$$

Proof of Theorem 3.2. At first we consider the case of $d=2 l+1(l=1,2, \ldots)$. Put

$$
\begin{aligned}
& I_{2}=\sup _{t}\left|P\left(n^{d / 2}\left(Y_{n}-\theta\right) \leq t\right)-P\left(\sum_{j=0}^{l} \tau_{j} \leq t\right)\right| \\
& =\sup _{t}\left|P\left(\sum_{j=0}^{l} T_{j, n}+R_{1 n} \leq t\right)-P\left(\sum_{j=0}^{l} \tau_{j} \leq t\right)\right| .
\end{aligned}
$$

Then we have

$$
I_{2} \leq I_{21}+I_{22}
$$

where

$$
I_{21}=\sup _{t}\left|P\left(\sum_{j=0}^{l} T_{j, n} \leq t\right)-P\left(\sum_{j=0}^{l} \tau_{j} \leq t\right)\right|
$$

and

$$
I_{22}=\sup _{t}\left|P\left(\sum_{j=0}^{l} T_{j, n}+R_{1 n} \leq t\right)-P\left(\sum_{j=0}^{l} T_{j, n} \leq t\right)\right| .
$$

Since $E T_{j, n}^{2}=O(1), j=0,1, \ldots, l$ and $E R_{1 n}^{2}=O\left(n^{-1}\right)$, applying (3.7) to $I_{22}$ we have

$$
I_{22}=O\left(n^{-\frac{1}{2}}\right)
$$

By (1.7) we can write $T_{j, n}, j=0, \ldots, l$ of the right-hand side of (2.5) as

$$
T_{j, n}=c_{1}\left(1-\frac{c_{2}}{n}+O\left(\frac{1}{n^{2}}\right)\right) n^{\frac{d-2 j}{2}}\left(U_{n}^{(k-j)}-\theta\right), \quad j=0, \ldots, l
$$


where $c_{1}(>0)$ and $c_{2}$ are generic constants. By Lemma 3.3 we have

$$
I_{21} \leq \sum_{j=0}^{l} \sup _{t}\left|P\left(T_{j, n} \leq t\right)-P\left(\tau_{j} \leq t\right)\right| .
$$

Using the relation $E\left|U_{n}^{(k-j)}-\theta\right|^{2}=O\left(n^{-(d-2 j)}\right), j=0, \ldots, l$ (see p.185 of Serfling,1980) and applying (3.1), (3.6) and (3.10) to the right-hand side of the above inequality, we have

$$
I_{21}=O\left(n^{-\frac{1}{2}}\right) .
$$

Because of (3.9) and (3.11), we get from (3.8)

$$
I_{2}=O\left(n^{-\frac{1}{2}}\right)
$$

which is (3.3).

Next we consider the case of $d=2 l(l=1,2, \ldots)$. On the right-hand side of (2.6), $T_{l-1, n}$ converges in distribution to $\tau_{l-1}$ and $T_{l}$ converges in probability to $\alpha_{l}$. We shall compute

$$
\begin{aligned}
& I_{3}=\sup _{x}\left|P\left(T_{l-1, n}+T_{l, n} \leq x\right)-P\left(\tau_{l-1}+\alpha_{l} \leq x\right)\right| \\
& =\sup _{x}\left|P\left(T_{l-1, n}+T_{l, n}-\alpha_{l} \leq x\right)-P\left(\tau_{l-1} \leq x\right)\right| .
\end{aligned}
$$

It follows that

$$
I_{3} \leq I_{31}+I_{32}
$$

where

$$
I_{31}=\sup _{x}\left|P\left(T_{l-1, n}+T_{l, n}-\alpha_{l} \leq x\right)-P\left(T_{l-1, n} \leq x\right)\right|
$$

and

$$
I_{32}=\sup _{x}\left|P\left(T_{l-1, n} \leq x\right)-P\left(\tau_{l-1} \leq x\right)\right| .
$$

For $I_{32}$, by $(3.2),(3.6)$ and (3.10) with $j=l-1$ we have

$$
I_{32}=o\left(n^{-\frac{1}{2}}\right) \text {. }
$$

Next we consider $I_{31}$. By (3.7), we have

$$
I_{31} \leq 4 E\left|T_{l-1, n}\left(T_{l, n}-\alpha_{l}\right)\right|+4 E\left|T_{l, n}-\alpha_{l}\right|
$$

where

$$
\begin{gathered}
T_{l, n}-\alpha_{l}=\frac{d(k, k-l) k !}{(k-l) ! d(k, k)}(A+B) \\
A=\frac{d(k, k)}{D(n, k) k !} n^{(k-l)} n^{l} U_{n}^{(k-l)}-E U_{n}^{(k-l)} \text { and } B=\left[1-\frac{d(k, k)}{D(n, k) k !} n^{(k-l)} n^{l}\right] \theta .
\end{gathered}
$$

By (1.7), we have

$$
A=U_{n}^{(k-l)}-E U_{n}^{(k-l)}+\left[\frac{c}{n}+O\left(\frac{1}{n^{2}}\right)\right] U_{n}^{(k-l)} \text { and } B=O\left(\frac{1}{n}\right)
$$


for some constant $c$. Because of $E\left[U_{n}^{(k-l)}-E U_{n}^{(k-l)}\right]^{2}=O\left(n^{-1}\right)$ (see, for example, Lee, 1990 , p.21), we have $E|A|=O\left(n^{-1 / 2}\right)$. Thus we get $E\left|T_{l, n}-\alpha_{l}\right|=O\left(n^{-1 / 2}\right)$ and $E\left|T_{l-1, n}\left(T_{l, n}-\alpha_{l}\right)\right|=O\left(n^{-1 / 2}\right)$. Thus by (3.13) we have $I_{31}=O\left(n^{-\frac{1}{2}}\right)$. From this and (3.12), we get

$$
I_{3}=O\left(n^{-\frac{1}{2}}\right)
$$

Since $E T_{j, n}^{2}=O(1), j=0,1, \ldots, l$ and $E R_{2 n}^{2}=O\left(n^{-2}\right)$, by the similar discussion to the first part of the proof in case of $d=2 l+1$ we have

$$
\begin{gathered}
I_{4}=\sup _{t}\left|P\left(n^{d / 2}\left(Y_{n}-\theta\right) \leq t\right)-P\left(\sum_{j=0}^{l-1} \tau_{j}+\alpha_{l} \leq t\right)\right| \\
\leq \sup _{t}\left|P\left(\sum_{j=0}^{l-1} T_{j, n}+T_{1 n} \leq t\right)-P\left(\sum_{j=0}^{l-1} \tau_{j}+\alpha_{l} \leq t\right)\right|+O\left(n^{-1}\right) .
\end{gathered}
$$

By (3.5), we have

$$
\begin{aligned}
I_{4} & \leq \sum_{j=0}^{l-2} \sup _{t}\left|P\left(T_{j, n} \leq t\right)-P\left(\tau_{j} \leq t\right)\right|+I_{3}+O\left(n^{-1}\right) \\
& =\sum_{j=0}^{l-2} \sup _{t}\left|P\left(T_{j, n} \leq t\right)-P\left(\tau_{j} \leq t\right)\right|+O\left(n^{-\frac{1}{2}}\right) .
\end{aligned}
$$

By the similar discussion to the case of $d=2 l+1$, by (3.2), (3.6) and (3.10) we have

$$
I_{4}=O\left(n^{-\frac{1}{2}}\right)
$$

which is (3.4).

In particular, for the case of $k=2$ and $d=2$ the rate of convergence of the Ustatistic is given by Theorem 6.5.1 of Borovskikh (1996) and Theorem 6.6.2 of Koroljuk and Borovskich (1994) as follows: Suppose the condition (A) that for S-operator

$$
S: f \rightarrow E\left[g\left(X_{1}, X_{2}\right) f\left(X_{2}\right) \mid X_{1}=x\right],
$$

there exists infinitely many indices $j$ for which the eigenvalues $\lambda_{j}$ of this operator $S$ are nonzero. Furthermore we assume that $E\left|g\left(X_{1}, X_{2}\right)\right|^{3}<\infty$. Then

$$
\sup _{-\infty<t<\infty}\left|P\left(n\left(U_{n}-\theta\right) \leq t\right)-P\left(\tau_{0} \leq t\right)\right|=o\left(n^{-\frac{1}{2}}\right),
$$

where

$$
\tau_{0}=\sum_{j=1}^{\infty} \lambda_{j}\left(Z_{j}-1\right)
$$

and $Z_{1}, Z_{2}, \ldots$ are independent standard normal random variables. In the proof of Theorem 3.2, we consider the case of $d=2 l$ and $l=1$. By (3.6),(3.10) and (3.14), we have $I_{32}=o\left(n^{-\frac{1}{2}}\right)$. Under the condition $E\left|g^{(1)}\left(X_{1}, X_{1}\right)\right|^{2}<\infty$, we have $I_{31}=O\left(n^{-\frac{1}{2}}\right)$. Hence we get $I_{3}=O\left(n^{-\frac{1}{2}}\right)$. Thus we have the following proposition. 
Proposition 3.4. Suppose that the condition (A), $E\left|g\left(X_{1}, X_{2}\right)\right|^{3}<\infty$ and $E \mid$ $\left.g^{(1)}\left(X_{1}, X_{1}\right)\right|^{2}<\infty$. Then we have,

$$
\sup _{-\infty<t<\infty}\left|P\left(n\left(Y_{n}-\theta\right) \leq t\right)-P\left(\tau_{0}+\alpha_{1} \leq t\right)\right|=O\left(n^{-\frac{1}{2}}\right),
$$

where $\tau_{0}=J_{2}\left(\xi_{2,2}\right)$ and $\alpha_{1}=2[w(2 ; 2) / w(1,1 ; 2)] \cdot\left[E g\left(X_{1}, X_{1}\right)-\theta\right]$.

Specially, for the LB-statistic $B_{n}$, by (2.13) we have

$$
\sup _{-\infty<t<\infty}\left|P\left(n\left(B_{n}-\theta\right) \leq t\right)-P\left(\tau_{0}+2\left[E g\left(X_{1}, X_{1}\right)-\theta\right] \leq t\right)\right|=O\left(n^{-\frac{1}{2}}\right) .
$$

For the V-statistic $V_{n}$, by $(2.103$ we have

$$
\sup _{-\infty<t<\infty}\left|P\left(n\left(V_{n}-\theta\right) \leq t\right)-P\left(\tau_{0}+E g\left(X_{1}, X_{1}\right)-\theta \leq t\right)\right|=O\left(n^{-\frac{1}{2}}\right) .
$$

For the statistic $S_{n},(3.16)$ holds with $S_{n}$ instead of $V_{n}$ in the first $P$ on the left-hand side. For the V-statistic, Theorem 6.6.1 of Koroljuk and Borovskich, 1994 shows that the rate of convergence is $o\left(n^{-1 / 2}\right)$ under the condition (A), $E\left[\left|g\left(X_{1}, X_{2}\right)\right|^{3}\right]<\infty$ and $E\left[\left|g^{(1)}\left(X_{1}, X_{1}\right)\right|^{3 / 2}\right]<\infty$. This is stronger than our result given (3.16). The reason why our result is weaker is due to the evaluation $I_{31}=O\left(n^{-1 / 2}\right)$.

\section{Acknowledgement}

The authors wish to express their thanks to the referee for his careful reading and kind comments.

\section{References}

Borovskikh, Yu.V. (1996). U-statistics in Banach spaces. VSP, Utrecht.

Kawata, T. (1972). Fourier analysis in probability theory. Academic Press, New York.

Koroljuk, V.S. and Borovskich, Yu.V. (1994). Theory of U-statistics, Kluwer Academic Publishers, Dordrecht.

Lee, A. J. (1990). U-statistics, Marcel Dekker, New York.

Nomachi, T., Kondo, M. and Yamato, H. (2001). Higher order efficiency of linear combinations of U-statistics as estimators of estimable parameters, Scientiae Mathematicae Japonicae, 56, 95-106.

Serfling, R. J. (1980). Approximation theorems of mathematical statistics. John Wiley, New York.

Shorack, G. R. (2000). Probability for statisticians. Springer, New York.

Toda, K. and Yamato, H. (2001). Berry-Esseen bounds for some statistics including LB-statistic and V-statistic, J. Japan Statist. Soc. 31, 225-237.

Yamato, H. and Toda, K. (2001). Asymptotic distributions of LB-statistics and Vstatistics for degenerate kernel, Bulletin of Informatics and Cybernetics, 33, 27-42. 
Yamato, H. , Kondo, M. and Toda, K. (2001). Asymptotic properties of linear combinations of U-statistics with degenerate kernels, (Submitted)

Received March 11, 2002

Revised September 24, 2002

Re-revised October 10, 2002 